\title{
A descriptive study of race and gender differences in how instructional style and perceived professor care influence decisions to major in STEM
}

\author{
Katherine Rainey ${ }^{1 *} \mathbb{D}$, Melissa Dancy ${ }^{1}$, Roslyn Mickelson², Elizabeth Stearns ${ }^{2}$ and Stephanie Moller ${ }^{2}$
}

\begin{abstract}
Background: Women and students of color are widely underrepresented in the majority of STEM fields. In order to investigate this underrepresentation, we interviewed over 200 male and female college seniors, primarily women and people of color, who either majored in STEM or started but dropped a STEM major. Here, we focus on one section of the longer interview that focused on students' perceptions of professor care as well as perceived and preferred instruction style. Additionally, we look at correlations between professor care, course interactivity, and sense of belonging. In our analysis, we examine student responses through the lens of gender, race, and their intersections.
\end{abstract}

Results: We found that white women perceived their STEM professors cared about them and their learning the most while women of color reported the least perceived care. Notably, men, regardless of race, reported similar perceptions of professor care. We found that students commonly report their STEM courses were lecture-based but say they would prefer more active approaches. In particular, we found that women who left STEM majors reported more lecture-based instruction while stating the highest preference for active learning environments. We found that perceiving their professors cared was related to a greater sense of belonging in STEM. Additionally, we found that students who reported active classrooms also reported more professor care in their STEM field.

Conclusions: Our findings indicate that active teaching environments may positively impact students' sense of belonging and desire to continue in STEM and that this impact may be higher for underrepresented students.

Keywords: Gender, Race, Representation, Course interactivity, Professor care, Belonging

\section{Introduction}

It is well-documented that women and students of color are underrepresented in most STEM fields (Holman et al. 2018; Hrabowski 2011; National Science Foundation, National Center for Science and Engineering Statistics 2017; United Nations Educational, Scientific and Cultural Organization 2015). To address this persistence of underrepresentation, many researchers have turned their attention to possible contributions of contemporary STEM pedagogy and curricula. Over the last few decades, studies

\footnotetext{
* Correspondence: Katherine.Rainey@colorado.edu

1Department of Physics, University of Colorado Boulder, UCB390, Boulder, CO 80309-0390, USA

Full list of author information is available at the end of the article
}

have shown benefits of incorporating pedagogies and curricula informed by research on student learning, including learning gains (Deslauriers et al. 2011; Terenzini et al. 2001), retention (Watkins and Mazur 2013), and improved attitudes toward science (Tseng et al. 2013). These outcomes often have disproportionate benefits for underrepresented students (Haak et al. 2011), which could point to consequences for increasing representation of women and people of color in STEM. The successful strategies share a common element: they move away from passive, lecturebased teaching toward more active approaches.

While there is a massive body of literature on the impacts of research-based teaching strategies and a separate corpus of research on impacts of gender and race in 
the STEM classroom, there is little overlap in the two bodies of research. The question of how the use of active approaches might influence women and underrepresented minorities (URMs) differently from men and white students is largely unanswered.

In this paper, we analyze in-depth interviews with a diverse set of undergraduate students in order to add to understandings about the intersections of teaching style in STEM with race and gender. Specifically, we consider students' perceptions of their college STEM classroom environments, their self-reported preferences for instructional style, and perceptions regarding whether their instructors care about their learning. Additionally, we look at the overlap of instruction style, perceived professor care, and sense of belonging, a factor that has been shown to impact persistence (Strayhorn 2012). In our analysis, we reject the deficit model of underrepresentation, which attributes underrepresentation to things students lack (e.g,. resources, academic support, exposure) and asserts representation can be achieved mainly by providing something to students, such as workshops and tutoring (Dancy et al. 2016). Here, we move toward a model focusing on systems students are embedded in-a classroom-focused approach for improving representation and equity in STEM.

\section{Prior research}

\section{Active teaching}

Research-based pedagogical approaches largely incorporate active techniques to engage students in their own learning. Active techniques take many forms, ranging from use of electronic clickers and peer discussion in large courses, to flipped classrooms and studio-style learning environments. To summarize a vast range of instructional styles, active learning can be thought of as "anything course-related that all students in a class section are called upon to do other than simply watching a lecture and taking notes" (Felder and Brent 2009).

\section{Impact of active teaching on learning and persistence for women and URMs}

There is robust body of research indicating that active teaching methods improve the learning of students (Freeman et al. 2014; Prince 2004). Many works, as mentioned above, show differential benefits of active learning for students from underrepresented groups, while a few others point to the opposite conclusion (Johnson et al. in press; Cabrera et al. 2001).

One extensive effort at addressing the impact of interactive vs. lecture-based teaching on underrepresented groups was undertaken by Madsen et al. (2013). They report on a meta-analysis of 26 published studies on the gender gap on concept inventories in physics. They found mixed results regarding impact of teaching style, with interactive methods having positive, negative, or neutral impacts on the gender gap depending on the study. Madsen et al. did not attempt to analyze the type of classroom beyond the self-reported status given by the authors of each study. We suspect that the wide variation in type of teaching associated with the ideas about "interactive," "reformed," "research-based," or "student-centered" may contribute to the mixed results. In other words, there is a range of how "interactive" teaching is implemented and the specifics of the implementation impact the results. Their findings indicate that active teaching can improve learning outcomes for women beyond that of men but likely depend on the nature of the implementation.

The most robust study of an implementation of a research-based, highly interactive pedagogy of which we are aware is reported by Beichner et al. (2007). They report results from a comparison of highly interactive studio-style classrooms to traditional lecture-based classrooms involving 15,000 students. They found all students were more likely to pass the class in the studio-style classrooms than students in the lecture-based classrooms. Notably, they also found that passing rates for women and URMs were improved more than those of men and white students.

In contrast, other studies have shown that there may be obstacles embedded in these approaches for students of certain identities. Gender and racial stereotypes can be upheld in group settings, which can inhibit participation from students with particular identities who fear they may uphold stereotypes about one or more groups they belong to (Steele et al. 2002). Further, the "chilly climate" encountered by minorities at predominately white institutions can permeate the science classroom (Cabrera 2001), likely making URM students more apprehensive to engage in active learning environments.

Despite these factors, active learning pedagogies may hold promise in addressing underrepresentation. Several other studies have attempted to disentangle the benefits of various instruction styles for students of different genders and races (Ballen et al. 2018; Cotner and Ballen 2017; Haak et al. 2011). However, the current literature has much room for growth and leaves open the question of if and how research-based teaching styles may differentially impact students of different genders and races. Our qualitative data address this open question.

\section{Intersectionality}

The analysis in this paper takes an intersectional approach. Intersectionality refers to the idea that aspects of one's identity (e.g., race, gender, class, sexual orientation) are not mutually exclusive, but instead interact to construct one's identity (Crenshaw 1991; Collins 2015). For example, considering race and gender as single axes of identity without analysis of the intersections of those groups can lead to the erasure of some identities, such as 
those of women of color (Bowleg 2008; Crenshaw 1991). In this study, we look at two axes of identity-race and gender-and the intersections of these identities: white men, white women, men of color, and women of color. Unfortunately, due to low numbers when parsing out by certain identities, not all analyses are intersectional in this same manner. We acknowledge this limitation but believe that this partial-intersectional approach will help avoid the erasure of some students' complex identities, such as those of women of color, by allowing the reader to see both gender and race analyses. Though there are other axes beyond race and gender that could be considered, these are the only axes of identity we recorded for this study.

\section{Methods}

\section{Interview data}

We report findings from in-depth interviews collected as part of a larger mixed method study, the Roots of STEM Success Project (https://clas-pages.uncc.edu/rootsofstem/). For other analyses using this data set, see (Mickelson et al. 2015; Moller et al. 2015; Rainey et al. 2018). The 2018 paper, which focuses on student sense of belonging, is of particular interest-the last few analyses of this manuscript build upon the results presented there. The Roots of STEM Success Project was designed to study experiences in STEM of students who are traditionally underrepresented in STEM fields. The Roots project includes a large quantitative dataset with middle school through college graduation administrative data related to STEM success of students who graduated from North Carolina high schools in 2004 and matriculated to 1 of the 16 campuses of the University of North Carolina (UNC) system. In addition to the quantitative data, in 2013, the Roots Project conducted 317 interviews with college seniors who were asked to reflect upon their family, community, secondary school, and college experiences related to their decisions to major in STEM fields. In this paper, we focus on a subset of 201 of those interviews that were conducted with students who were either majoring in STEM fields or had declared a STEM major and left it for a non-STEM field.

We identified prospective interviewees by distributing an email recruitment survey to seniors at all $16 \mathrm{UNC}$ campuses in January 2013. On these surveys, students could designate up to three major fields of study. Based on student responses, we categorized 201 respondents as STEM majors and STEM leavers (those who began in STEM but later elected to switch to a non-STEM major). To define a STEM major, we use the categorization utilized by the National Science Foundation Advance Program (http://www.nsf.gov/crssprgm/advance/index.jsp) where majors such as engineering, physical sciences, earth, atmospheric or ocean sciences, mathematical and computer sciences, and biological and agricultural sciences are considered as falling within the STEM category. We excluded the social sciences in our categorization of STEM, as we are interested in gender underrepresentation. A student is categorized as a major if their current major, at the time of the interview, falls in STEM. Students are classified as STEM leavers if they originally majored in STEM and then changed their majors to a non-STEM field.

We restricted our sample to students who attended public school (K-12) in North Carolina and who were younger than 30 years of age. These selection criteria were designed to align the interview sample with the quantitative sample in the larger project. Students were asked to identify their racial/ethnic group on the survey. Once the potential interviewees were identified, we reached out to them via email to set up an interview (either via Skype, phone, or in person). We attempted to match interviewers with interviewees according to gender and race to reduce power differentials between interviewers and respondents (Seidman 2013), although this was not always possible. The interviews lasted between $30 \mathrm{~min}$ and $1 \mathrm{~h}$ and were recorded for transcription purposes. Students were paid US\$25 for participation in the interview.

\section{Interview sample}

In this article, we focus on interviews with 201 college seniors from the sampling frame of self-selected qualified volunteers. Because we oversampled students underrepresented in STEM, our purposive sample is not representative of STEM students in either race or gender. Of all students interviewed, 66\% identified as women and 34\% identified as men. Additionally, $48 \%$ of the students identified as white and 52\% identified as students of color: $31 \%$ black, $8 \%$ Asian, 7\% Hispanic, 2\% multiracial, and 1\% Native American. We selected respondents based on how they identified their racial or ethnic identity on the recruitment survey; we employ their self-identified race in reporting findings of this study.

Given that we are interested in how students' multiple and intersecting identities impact their STEM experience and how it helps us better understand STEM outcomes, we disaggregate the sample by race, gender, and status (major vs. leavers). This resulted in some categories with very small numbers (i.e., we only interviewed four Hispanic women who were majors). Therefore, we collapsed categories in order to report findings in meaningful ways. When we looked at responses across different racial and ethnic groups, we consistently noticed similar patterns. For instance, the responses of Hispanic students and black students looked more similar compared to the responses of white students. Therefore, we chose to form two racial groups based on representation of one's racial group in their field. Thus, we look at white students and URMs. Due to their complex identities as students of color who 
are well-represented in STEM, and relatively low numbers in our sample, we chose to exclude Asian students from our analysis.

\section{Interview protocol}

The interview protocol was developed specifically for the Roots of STEM Success Project. Questions were designed to elicit a recounting of the participants' history with STEM and factors that influenced their decision to major in STEM. We asked about factors such as family and peer influences, childhood informal educational experiences, secondary school and college experiences in and out of the classroom, beliefs about the self, attitudes toward STEM, and the students' reasons for pursuing or not pursuing a STEM major. The findings presented in this paper focus on two areas of the interview-one related to perceptions of professor care in STEM majors and one related to instruction style of their STEM major courses.

\section{Interview analysis}

We followed conventional procedures for analyzing the qualitative data captured in our interviews. Multiple members of the research team worked together to develop a coding rubric that was both prescriptive (based on ideas we knew we wanted to explore) and emergent (based on reiterative coding and discussion of a subset of interviews) in order to allow unexpected ideas to emerge. Through discussion, codes were compared, defined, reorganized, and collapsed until the final coding rubric was developed. Under this coding scheme, several broad codes related to perceived professor care, reported levels of preferred and experienced course interactivity and belonging were used to code passages for further, more detailed coding and analysis as elaborated on below as results are presented. Analysis and interpretation was done primarily by white women, with social and physical science backgrounds.

In this paper, we present graphical representations of student's responses extracted from the interviews. In addition, we report numerical measures of their statistical significance. This was done by making comparisons between two specific groups with dramatically different responses or one particular group that stands out with the rest of the respondents for a particular test. Using a test of proportions, we calculated Z-scores for various comparisons as follows:

$$
Z=\frac{\hat{p}_{1}-\hat{p}_{2}}{\sqrt{\hat{p}(1-\hat{p})\left(\frac{1}{n_{1}}+\frac{1}{n_{2}}\right)}},
$$

where $\hat{p}_{i}$ is the proportion of people giving the analyzed response in relation to the total number of people in that group; subscripts 1 and 2 label the groups being compared. The $\hat{p}$ without subscripts is the proportion of people giving the response in relation to the total number of people being compared and $n$ represents the sample size. In every case where we report significance, we have used this test of proportions and calculated significance from the $\mathrm{z}$-score. The z-score conveys the probability that respondents' responses to interview questions are statistically independent of their various demographic characteristics. We acknowledge the limitations to the use of quantitative analysis of qualitative data and the debatable use of statistical tests of qualitative data (Maxwell 2010).

\section{Results \\ Professor care in STEM}

In the interview, participants were asked: Do you think your \{major or dropped major\} instructors cared about you and your learning? We coded their answers broadly as either "instructor cared" or "instructor did not care." We simply report on students' perceptions of professor care and do not sub-code based on reasons given. Students who reported both having professors who cared and professors who did not care were counted twice, once for each response. For example, a math major may report that they had a calculus instructor who cared about their learning and a linear algebra instructor who did not care about their learning. Their responses would be counted twice, once for each code. We report our findings based on race and gender, as well as by the type of STEM field (i.e., biological sciences versus physical sciences).

We were able to analyze responses to the question about professor care for 135 majors and 38 leavers. Our data shows majors were significantly more likely than leavers to report a professor who cared about their learning $(p \leq 0.05)$, as shown in Fig. 1.

\section{Relationship of gender and race to perceived professor care} Analyses based on respondents' gender and race are presented in Fig. 2, for both majors and leavers. We found white women majoring in STEM were the most likely to report feeling cared about by their instructors while women of color were the least likely $(p \leq 0.05)$. Notably, women of color majoring in STEM report less care than the white women who dropped their STEM major. Men's responses, in contrast, did not vary significantly based on race, as highlighted in Fig. 2. We saw nearly identical results for both white men and men of color majoring in STEM, and only a small racial difference among male leavers. The findings indicate small variations in perceived professor care across gender by race cohorts. All leavers tend to perceive less care than majors of the same race and gender, and white female leavers reported as much care as male majors of any race. Women of color, whether majors or leavers, report less professor care than any other cohort. 


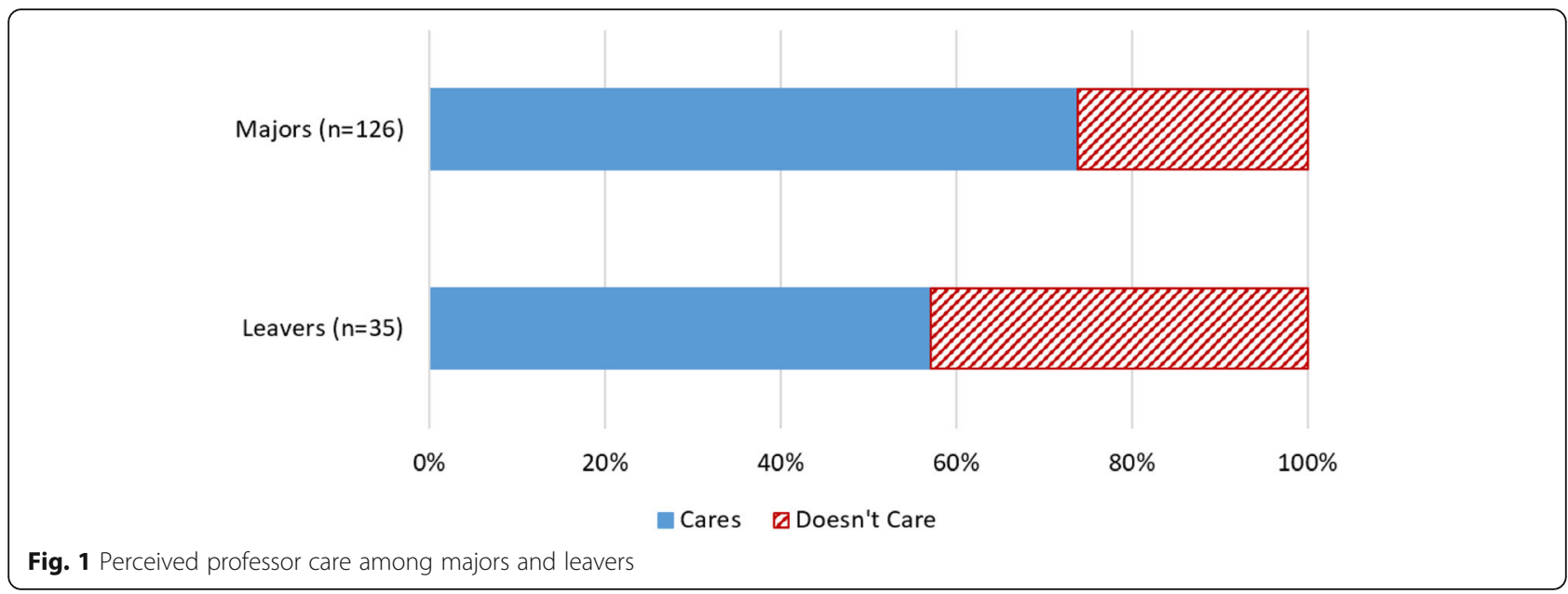

\section{Relationship of representation status to perceived professor} care

Perceptions of professor care were analyzed based on discipline, determined by gender representation in respective STEM fields-biological sciences are near parity with regards to gender representation whereas the physical sciences (pSTEM) are far below. As can be seen in Fig. 3, students in pSTEM fields were less likely than those in biological science fields to feel their professors cared about their learning. This difference appears for majors and leavers. Comparisons between pSTEM and biological science, combining majors and leavers, resulted in $p \leq 0.001^{*}$. It should be noted that these students' responses were frequently about STEM instructors in general and does not necessarily refer to perceptions of care solely within the major itself.

It is of note that this analysis was not done based upon racial representation within STEM fields due to the consistent low representation of people of color across all STEM fields.

\section{Summary of professor care}

Most students perceive that their professors care about their learning. However, across all demographic groups, majors are more likely than leavers to report feeling their professors cared about them and their learning. Among all majors, 75\% reported feeling their STEM professors cared about them and their learning. While it is not surprising that majors reported higher levels of care than leavers, it is worth noting that all of these students were seniors nearing graduation in their field. However, fully $25 \%$ of students about to graduate with a STEM major reported feeling their STEM professors did not care about their learning, which raises an issue of concern to be investigated further. While the results for white men and men of color were nearly identical, white

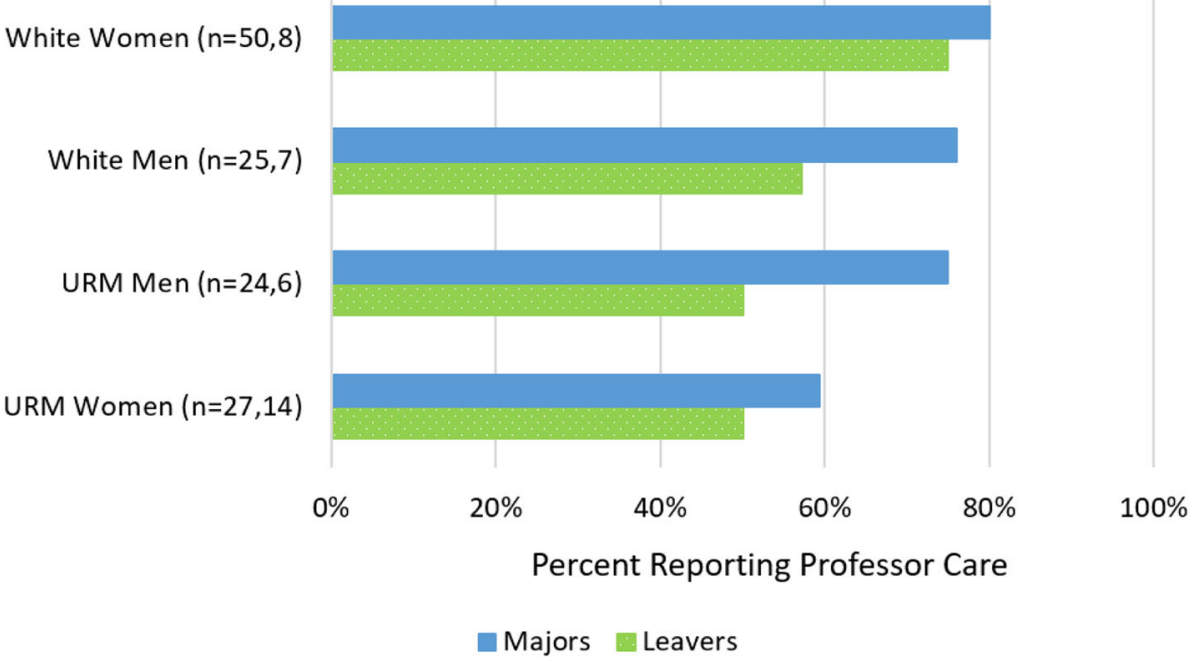

Fig. 2 Perceived professor care by major status, race, and gender. $N$ values are report for majors and leavers, respectively 


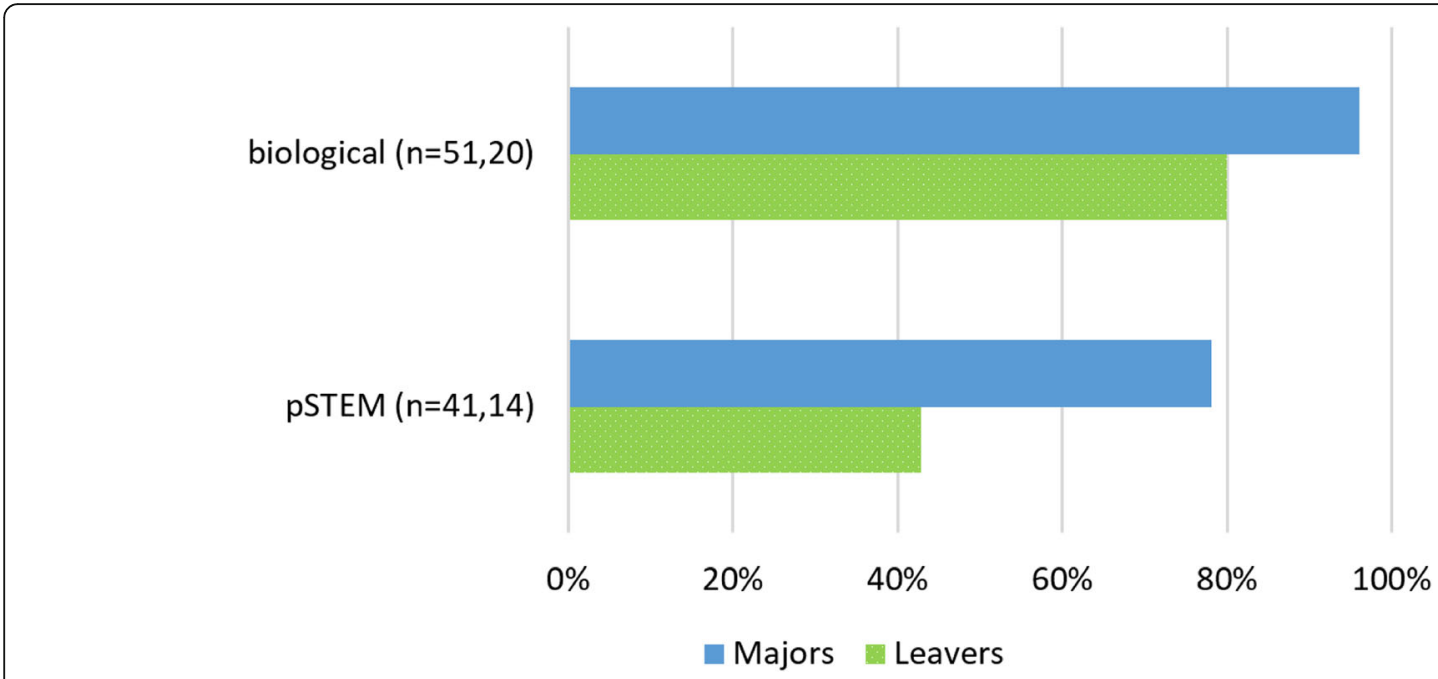

Fig. 3 Perception of professor care by field, biological sciences, and physics sciences (pSTEM)

women reported the most care while women of color reported the least. Only $60 \%$ of the women of color in our sample reported feeling their professors cared about them, despite being seniors about to receive their degrees in a STEM field.

Reasons given by women of color for not feeling their professor cared varied. A common reason was poor or distant teaching. For example, a black female leaver stated:

I felt like they were there just to teach us what we were supposed to know and if we didn't grasp it then it wasn't their problem. I would get outside tutoring help and they would try to help me out as best as I could but because my teacher wasn't teaching the basics it was just hard for me to grasp onto the concepts.

Large class sizes were also a common reason students gave for feeling lack of care. A multiracial female major noted:

I didn't feel they [cared] because there are so many kids in the class. I think it's hard to really connect with your teachers.

Unfriendly interactions with individual teachers were also frequently mentioned. For example, one women told a long and involved story of feeling unsupported by her professor after returning to school after a family death.

The extent to which students, particularly women of color, feel their professors care about them and their learning may influence their persistence, though we cannot infer direct causation. We do not question whether the vast majority of faculty care greatly about their students and their learning, and we know of no research-based evidence to the contrary. However, our interviews suggest that many students do not feel this care. We note that it is not unusual for introductory science courses to have large enrollments where there is little opportunity for personal contact between the professor and students. Leavers are likely to have only taken these large-enrollment introductory classes (as opposed to smaller, upper-division classes), and this could provide a reasonable explanation for the differences in perceived professor care between majors and leavers-it could hold true that majors feel more professor care from their upper-division course professors. However, specific courses were not always discussed in the interviews, and thus we cannot concretely make this conclusion.

\section{Instruction style of STEM courses}

Our findings regarding instructional style of STEM courses are based on responses to two questions we asked all interviewees:

1. To what extent did your math and science teachers lecture vs. use more active approaches such as, encouraging student discussion, cooperative learning, and hands on activities?

2. Would you have preferred a different emphasis?

Responses to this question were coded based on the reported professors' instruction style and students' stated preference for instruction style in their STEM courses. Most students were not specific about the types of active or mixed approaches they encountered or preferred, making coding based largely on their own interpretations and definitions. This also means coding was straightforward and coders did not arrive at wildly different coding schemas during their separate then combined analyses. Student responses were coded in three ways: (1) active instructional 
style, (2) lecture-based instructional style, and (3) mixed instructional style. Responses indicating a mixed instructional style meant that some active methods were reported, but lecture was also highly present in the course. For the most part, when a student was coded for having mixed experiences, they reported that the main class was lecture-based but there was a lab component that was active. For example, a black female leaver stated,

You have the class and then you have the lab. The lab is the place for you to work hands-on with your classmates or whatever and then class is just lecture.

Sometimes, a student reported different experiences in different courses. For example, a white female major described her chemistry class

In the entry level chemistry courses where you have a larger class, most of the time the teacher ends up lecturing. But, once you get into the junior and senior level courses it's no longer so much concentrated on lecturing as it is on, 'here's some problems, this is the basic outline and how you should be thinking about these problems, now work together or work alone and help each other figure out these problems.'

In this situation, her response was coded as both active and lecture-based.

Students' preferences were coded as either desirous of a different emphasis or as comfortable with the reported approach to instruction. It is worth noting that all responses are based on student perceptions of interactivity and may not align with the instruction style professors thought they employed or with or the perceptions of other students in the same course.

\section{Perceived instruction style}

We report students' perception of the instruction style they encountered based on both gender and race. Results based on gender are shown in Fig. 4. Active instruction was the least frequently reported instruction style for all groups. Female leavers reported the lowest levels of active instruction. In contrast, female leavers reported lecture at higher rates than other groups $(p \leq 0.001)$. Male leavers and female majors reported similar instruction styles. Notably, male majors reported encountering mixed instruction styles more frequently than lecture-based instruction, whereas female majors did not have a significant difference in frequency of mixed or lecture-based instruction.

Results by race are shown in Fig. 5. Active instruction was the least frequently reported instruction style for all racial groups. URM leavers reported encountering active instruction at lower rates than other groups. In contrast, the number of students reporting lecture-based instruction was much larger than that of active teaching. The responses of leavers diverge substantially from those of majors for both mixed and lecture-based instruction. URM leavers were significantly more likely than other groups to encounter lecture-based instruction $(p \leq 0.05)$, though white and URM leavers reported lecture at comparable rates.

\section{Preferred instruction style}

We report student preferences for instruction style by gender in Fig. 6 and by race in Fig. 7. Of note, students generally report preferring more active-based teaching than they report encountering. Women leavers stand out among those with the greatest preference for active methods compared to other groups $(p \leq 0.05)$, despite reporting encountering that method in their STEM courses the least.

Data for instruction style preference based on race is presented in Fig. 7. White and URM majors had similar preferences for instruction style. Leavers, however, did not. Notably, URM leavers report the greatest preference for active teaching compared to other groups, whereas white leavers had the greatest preference for lecture-based instruction. It is striking that active approaches to instruction are the most popular while lecture-based instruction is the least favored approach among all students.

\section{Summary of instruction style}

We found a large discrepancy between preferred instruction style and reported instruction style. In particular, preferences for instruction style lean toward more active approaches. We note this preference for more active teaching is present across all demographic groups. We see that female leavers and leavers of color were the most likely to prefer active instruction styles but were the least likely to report experiencing it. This finding suggests that students from underrepresented groups who are leaving may have been affected by a mismatch in their preferred instruction style and the instruction styles they perceived in STEM courses. This may especially be true for women of color.

Because our data are qualitative, we cannot directly correlate teaching style to persistence. There is another study in STEM, which looked exclusively at calculus students, that concluded that those who do not persist are more likely to perceive their classrooms to be less interactive than students in the same classroom who persisted (Ellis et al. 2014). These findings may be consistent with ours. Leavers who report lecture-based teaching may not have experienced more lectures than their counterparts who report more active classrooms, but leavers perceive their classrooms to be less active. Our data indicate this is especially the case for underrepresented groups. Leavers from underrepresented groups report a greater preference for active instruction styles than majors of all demographics, but we cannot 


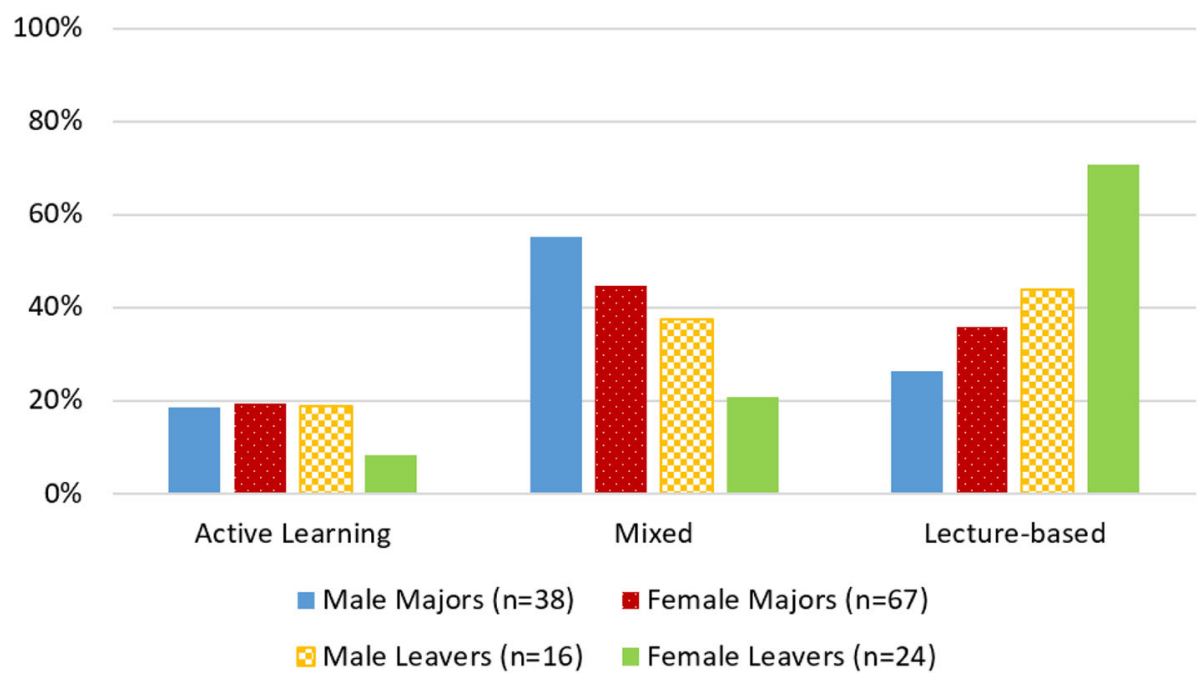

Fig. 4 Perceived instruction style by major status and gender

report that compared to other leavers or majors they experienced different classroom pedagogy. It is possible that students encountered similar pedagogical approaches but were disproportionately engaged by the instructor. Regardless, our findings suggest the potential positive impact of reforming teaching so it is more active on the retention of underrepresented groups. This is an area of potential importance for which more research is needed.

\section{Intersections of belonging, perceived professor care, and instructional style}

During the interview, students were also asked if they felt they belonged in their major or former STEM major (those who left the major). Alternatively, they may have brought up belonging on their own during the interview. Responses were coded as "belongs," "does not belong," or reports "mixed belonging" (if a student said they sometimes felt they belonged and sometimes did not). Further details on this analysis and presentation of results related solely to belonging are reported in another paper (Rainey et al. 2018). In this section, we consider the ways that sense of belonging, perceived professor care, and instructional style intersect for majors and leavers. Because not all interviews could be coded for all questions, the number of responses for the intersections analyses are often lower than other reports. Analysis of race, gender, and their intersections was unable to be conducted due to low numbers.

\section{Belonging and feeling professor care}

In this section, we report how interviewees' belonging responses related to the care they perceived from their professors. Findings are as shown in Fig. 8. Similar trends were seen for both majors and leavers, so the aggregated data are presented. We found that those who reported professor care were more likely to report feeling they belong in STEM than those who did not feel cared about $\left(p \leq 0.01^{*}\right)$.

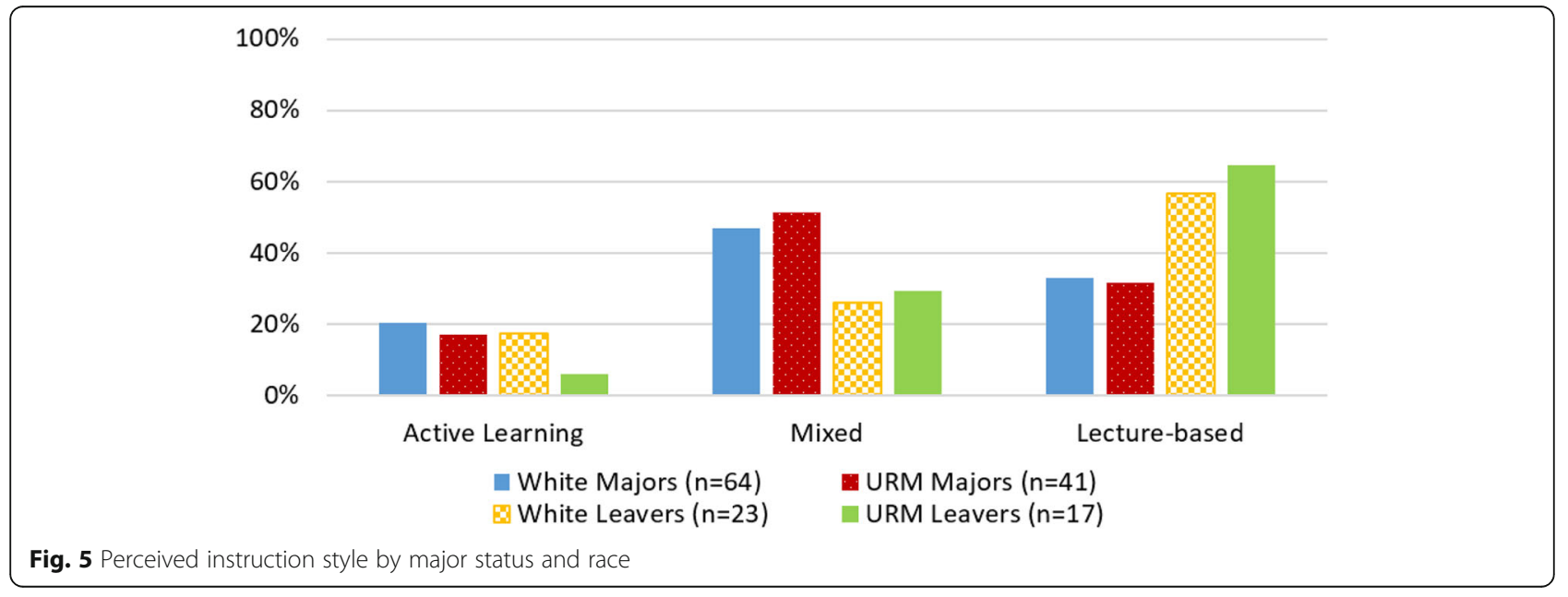




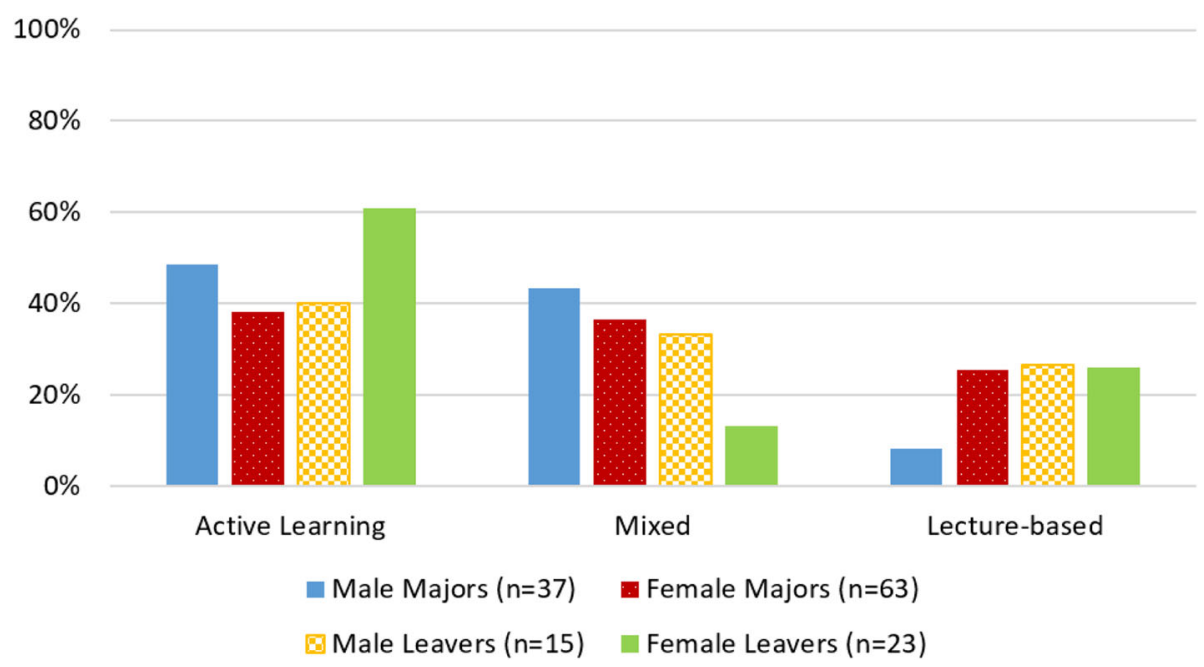

Fig. 6 Preferred instruction style by major status and gender

\section{Lecture-based instruction and decreased belonging}

Analysis was done to see how belonging and instruction style are connected. Our data, presented in Fig. 9, shows a slight increase in lack of belonging for those in lecture-based courses, though this trend is not statistically significant.

\section{Active learning and greater feelings of professor care}

Our final analysis looks at the connection between encountered instruction style and perceived professor care. We see a correlation between reported instruction style and perceived professor care: as the level of active learning teaching methods increases, students are more likely to report their professors care. Results appear in Fig. 10. Students perceived professors who employed active approaches as more caring than those who used lecture-based instruction $(p \leq 0.01)$.
Sense of belonging, caring professors, and active classrooms

Our data suggest a connection between students' perceptions of their professors' instruction styles, feelings of belonging in their major, and whether their professors care about their learning. We see distinct differences in sense of belonging among STEM majors who felt professors cared and those who did not. Those who felt professors did not care were far more likely to report feelings that they did not belong in their major. We also saw a relationship between instruction style and perceived professor care. Students who experienced active teaching methods were more likely to report feeling cared for by their professors. Moreover, we found gender and racial differences in these perceptions consistent with demographic patterns of underrepresentation in STEM.

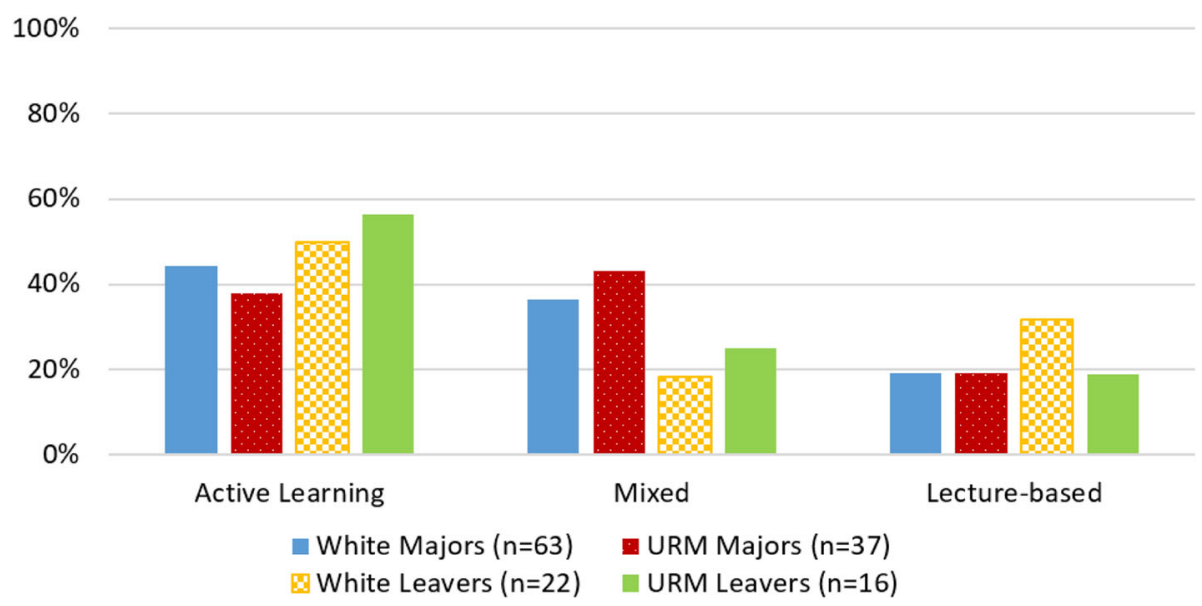

Fig. 7 Preferred instruction style by major status and race 


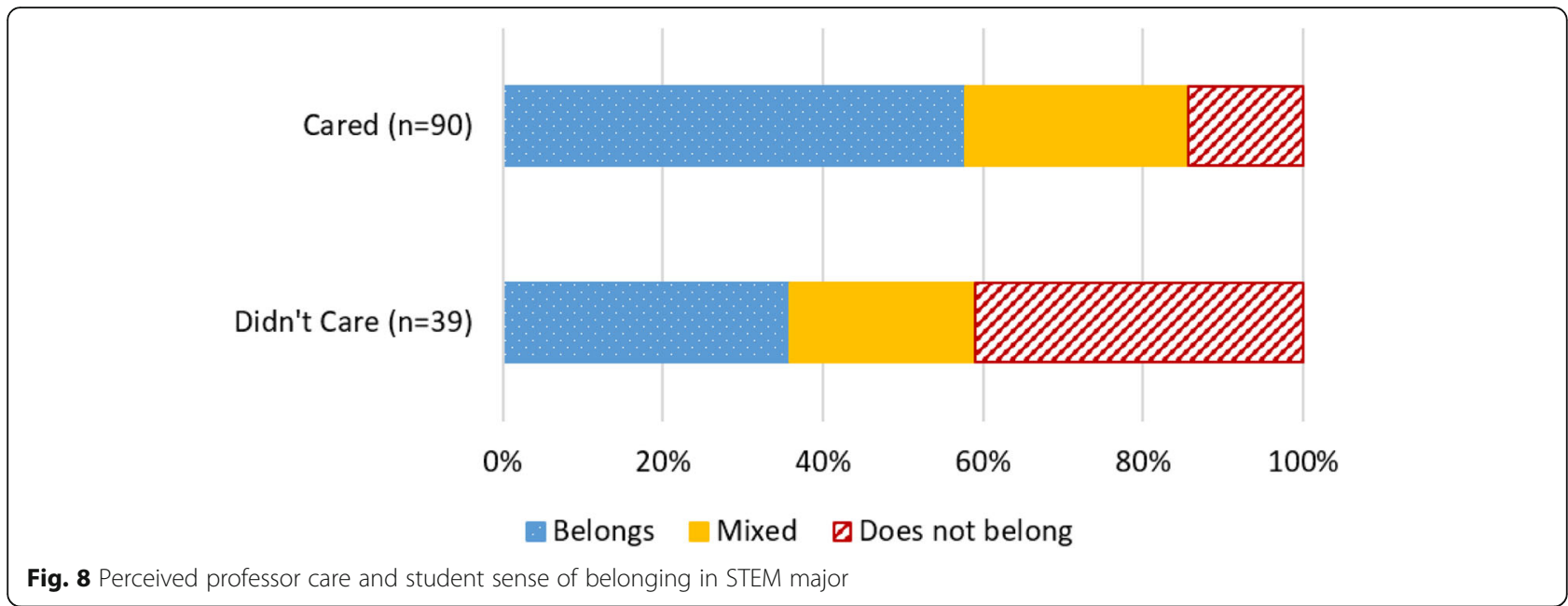

\section{Discussion}

Despite improving trends, women and people of color remain underrepresented in STEM fields. Findings from the growing corpus of research on the problem suggest many factors contribute to a complex dynamic that results in the underrepresentation of women and students of color in STEM. We advance understanding of the persistence of underrepresentation among women and students of color by analyzing interviews we conducted with 200 North Carolina college students who discussed the pedagogy they experienced in their STEM courses, whether they felt they belonged in these courses, and if they perceived their STEM professors cared about their learning. We compared STEM majors' perceptions of these three factors with perceptions of who left STEM for another discipline. We also made comparisons between men and women and students from underrepresented minority groups and white students. Our findings fall into six categories:
1. Majors are more likely than leavers to report feeling their professors cared about their learning.

2. Women of color, whether majors or leavers, perceive less professor care than students from any other gender/race cohort.

3. Students in a pSTEM field are less likely to report feeling their STEM professors cared about them and their learning than those in biological sciences.

4. Women who left STEM evidence the strongest preference for active approaches but experience it least frequently in their STEM courses.

5. Students who feel they belong in STEM are more likely than students who did not feel they belonged to also report their professors cared about them.

6. Students who recount encountering more active approaches also feel more cared about by their professors.

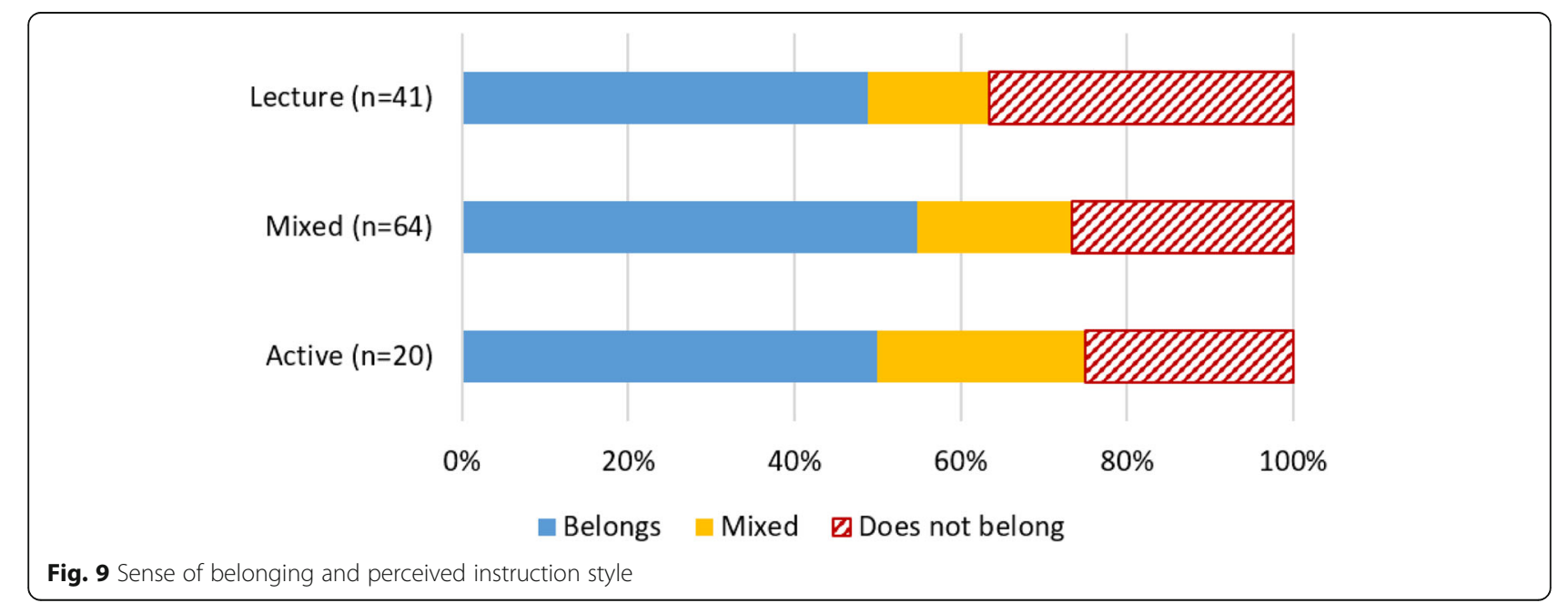




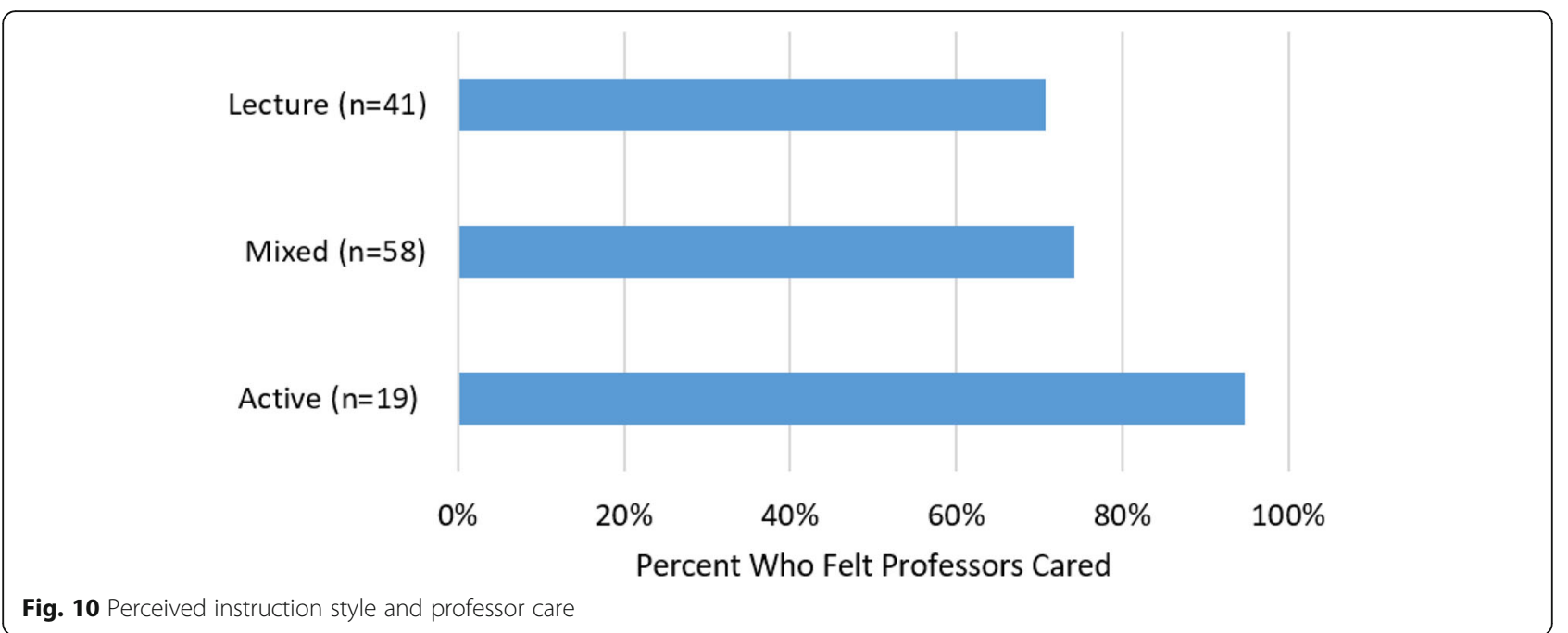

While we cannot make any causal claims, together these findings shed needed light on the persistent demographic underrepresentation of women and students of color in most STEM fields. Our study is unique in several ways that go beyond other findings, suggesting that successful teaching strategies should move away from passive, lecture-based teaching toward active approaches that benefit all students. First, we consider race and gender and their intersections in contrast to most studies that consider only one dimension or the other. Secondly, our data come from a self-selected sample of college students with a wide range of backgrounds who attended 1 of the 16 public universities in North Carolina. Many studies report data from only one institution or from more selective institutions. Third, we consider differences across different STEM fields instead of looking at only a single field or collapsing all majors into a single STEM category. Fourth, we consider perceived impacts of instructional style on persisting in the STEM major, a topic that is rarely studied. Fifth, we connect perceived belonging and whether the students perceive their professors as caring if they learn to the issues of instructional approaches in STEM. To date, no other investigations of STEM majors has incorporated these disparate elements of the wider social and educational context to the underrepresentation of women and people of color.

The ultimate question for researchers in this area is "What can be done to increase the representation of women and people of color in STEM?" Therefore, we consider what our work contributes toward the answers to this very big question. In order to frame our recommendations, we first offer a friendly critique of typical change efforts and illuminate an alternative model.

While rarely explicitly stated, many change efforts around equity operate under a deficit model of change. The deficit model (Green 2006; Meyer Monhardt 2000) views the problem through the lens of individuals instead of larger cultural systems. Under the deficit model, it is assumed that women and people of color lack something (academic preparation, social capital, role models, assertiveness, confidence, experiences, encouragement, mathematical skills, etc.) that hinders their participation in STEM. By providing supplements (i.e., extra training and opportunities for experience, scholarships, mentoring, etc.), the problem can be addressed. Under this model, gender and ethnic/racial equity in STEM will be reached when those who are marginalized (women and people of color) change to fit the system that is in place. This model does little to question the culture or structures that contribute to the marginalization of the groups are being asked to adapt to the extant system of STEM education.

This model is flawed because it fails to recognize larger cultural systems and places responsibility for change on those who are marginalized, while it leaves intact the cultural climate, the organizational structures, and classroom practices that help create the problem in the first place. Undoubtedly, efforts to support the academic experiences of women and people of color can be valuable; they may not be sufficient for significant and lasting change to occur. Decades of such programmatic interventions built upon the deficit model have had only minor successes.

In contrast to the deficit model of change is a systems model of change. Under the systems model, it is the environmental and cultural structures that are the target units for change. Structures within the system can act to privilege some and thereby disadvantaging others. The deficit model can be thought of as a "fix the people" model, whereas the systems model is a "fix culture and systems" model. Our findings support a need to view change through a systems model. Specifically, our interviews suggest that the classroom environment likely is experienced differently by those students from underrepresented groups than 
students from more privileged gender and ethnic backgrounds. While all students found an association between perceptions of a classroom as active and perceptions that their professors cared about their learning, women and students of color who leave STEM are the most likely to report a disconnection between perceived classroom environment and what they desire. Further, women of color in our sample were significantly less likely to feel their professors cared about them.

Taken together, our findings suggest that a fruitful way to address representation issues is to address classroom environments. Recent surveys indicate that while there is a growing trend toward more active teaching approaches, the majority of faculty report extensive lecturing. For example, the 2010-2011 HERI survey of faculty (Hurtado et al. 2012) found that, in STEM, the majority of faculty ( $70 \%$ of male faculty and $50 \%$ of female faculty) report using extensive lecture most or all the time. Surveys in individual disciplines have found similar results. A 2010 survey of calculus faculty found $80 \%$ of instructors lecturing "very often" or "often" (Apkarian and Kirin 2017). The majority of STEM faculty use extensive lectures the majority of the time. Our results indicate that shifting toward more active approaches may increase the persistence of women and students of color.

We note that as teaching becomes more active, and less lecture-based, the level of interpersonal interactions both between students and the student and their professor increases. We expect that as meaningful interpersonal interactions increase so will feelings of care from faculty, which can support a greater sense of belonging, especially among marginalized students.

\section{Conclusions}

Women and students of color continue to be underrepresented in most STEM disciplines. Several studies have investigated curriculum and pedagogical approaches as possible explanations for representation discrepancies in STEM. In this study, we focus on preferred and encountered active-teaching levels in STEM courses and students' perceptions of professor care. We analyzed this data in relation to students' gender and race, as well as students' status as a STEM "major" or "leaver." Additionally, we looked at the intersections of course interactivity, professor care, and student sense of belonging in STEM.

Using a series of interviews with undergraduates from diverse ethnic backgrounds, we sought to gain greater understanding of why there is persistent underrepresentation of women and students of color in STEM majors. Our findings suggest a discrepancy in the preferred and encountered instruction style for underrepresented students-in particular women and underrepresented minorities who left their STEM majors-who preferred more active approaches. Additionally, we found that women of color were the least likely to feel their STEM professors cared about their learning. A sense of professor care was associated with active learning courses and a higher sense of belonging in STEM. Though our data are correlational, they point to the notion that increasing use of activeteaching approaches is a possible way to support students underrepresented in STEM and increase retention of those students.

The underrepresentation of women and students of color in STEM is a complex problem with roots in many institutions and cultural experiences that students faced prior to entering higher education. While we fully acknowledge the importance of these prior encounters, the findings of this paper focus on how certain features of the organization and culture of STEM disciplines contribute to the problem as well. Our findings further underscore the necessity of taking a systems approach to change, rather than attempting to equip individual students from marginalized groups better to withstand aspects of the culture of the STEM disciplines that they-as well as some students from more privileged groups-find problematic. Then, problems with underrepresentation may be ameliorated.

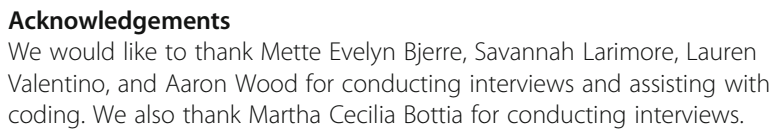

\section{Funding}

The authors gratefully acknowledge support provided by the National Science Foundation award numbers DUE-0969286, DRL-1420363, and DRL-1420350. Any opinions, findings, and conclusions or recommendations expressed in this material are those of the authors and do not necessarily reflect the views of the National Science Foundation.

\section{Availability of data and materials}

Data was collected via interviews which could be personally identifiable. Sharing of collected data was not approved by the Institutional Review Board.

\section{Authors' contributions}

$\mathrm{MD}$ contributed to the conception and design of the study; led the initial draft of the data codebook; participated in data collection, analysis, and interpretation; and led writing and revising of the manuscript. KR contributed to the analysis and interpretation and led writing and revising of the manuscript. RM contributed to the conception and design of the study; contributed to data collection, analysis, and interpretation; and contributed to the writing and revising of the manuscript. ES contributed to the conception and design of the study; supervised execution of data collection; contributed to analysis and interpretation; and contributed to the writing and revising of the manuscript. SM contributed to the conception and design of the study; contributed to data collection, analysis, and interpretation; and contributed to the writing and revising of the manuscript. All authors read and approved the final manuscript.

\section{Ethics approval and consent to participate}

All interview participants gave informed consent prior to being interviewed. The University of North Carolina at Charlotte's Institutional Review Board approved all data collection under protocol \#13-0112.

\section{Consent for publication}

The manuscript does not include images or videos relating to individual participants.

Competing interests

The authors declare that they have no competing interests. 


\section{Publisher's Note}

Springer Nature remains neutral with regard to jurisdictional claims in published maps and institutional affiliations.

\section{Author details}

'Department of Physics, University of Colorado Boulder, UCB390, Boulder, CO 80309-0390, USA. ${ }^{2}$ Department of Sociology, University of North Carolina at Charlotte, 9201 University City Blvd, Charlotte, NC 28223, USA.

Received: 26 May 2018 Accepted: 15 January 2019

Published online: 01 February 2019

\section{References}

Apkarian, N., Kirin, D., Bressoud, D., Rasmussen, C., Larsen, S., Ellis, J., Ensley, D. and Johnson, E. (2017). Progress through calculus: Census survey technical report. Available at http://maa.org/ptc.

Ballen, C. J., Aguillon, S. M., Brunelli, R., Drake, A. G., Wassenberg, D., Weiss, S. L., et al. (2018). Do small classes in higher education reduce performance gaps in STEM? BioScience, 68(8), 593-600

Beichner, R. J., Saul, J. M., Abbott, D. S., Morse, J. J., Deardorff, D., Allain, R. J., et al. (2007). The student-centered activities for large enrollment undergraduate programs (SCALE-UP) project. Research-based Reform of University Physics, 1(1), 2-39.

Bowleg, L. (2008). When black+ lesbian+ woman \# black lesbian woman: the methodological challenges of qualitative and quantitative intersectionality research. Sex Roles, 59(5-6), 312-325.

Cabrera, A. F., Colbeck, C. L., \& Terenzini, P. T. (2001). Developing performance indicators for assessing classroom teaching practices and student learning. Research in Higher Education, 42(3), 327-352.

Collins, P. H. (2015). Intersectionality's definitional dilemmas. Annual Review of Sociology, 41, 1-20

Cotner, S., \& Ballen, C. J. (2017). Can mixed assessment methods make biology classes more equitable? PLoS One, 12(12), e0189610.

Crenshaw, K. (1991). Women of color at the center: Selections from the third national conference on women of color and the law: Mapping the margins: Intersectionality, identity politics, and violence against women of color. Stan. L. Rev, 43, 1241-1243.

Dancy, M. H., Rainey, K., Mickelson, R., Stearns, E., \& Moller, S. (2016). Influences of teaching style and perceived care of instructor on retention of underrepresented groups in STEM, 2016 PERC Proceedings. Sacramento.

Deslauriers, L., Schelew, E., \& Wieman, C. (2011). Improved learning in a largeenrollment physics class. Science, 332(6031), 862-864.

Ellis, J., Kelton, M. L., \& Rasmussen, C. (2014). Student perceptions of pedagogy and associated persistence in calculus. ZDM, 46(4), 661-673.

Felder, R. M., \& Brent, R. (2009). Active learning: an introduction. ASQ Higher Education Brief, 2(4), 1-5.

Freeman, S., Eddy, S. L., McDonough, M., Smith, M. K., Okoroafor, N., Jordt, H., \& Wenderoth, M. P. (2014). Active learning increases student performance in science, engineering, and mathematics. Proceedings of the National Academy of Sciences, 111(23), 8410-8415.

Green, D. (2006). Historically underserved students: what we know, what we still need to know. New Directions for Community Colleges, 2006(135), 21-28.

Haak, D. C., HilleRisLambers, J., Pitre, E., \& Freeman, S. (2011). Increased structure and active learning reduce the achievement gap in introductory biology. Science, 332(6034), 1213-1216.

Holman, L., Stuart-Fox, D., \& Hauser, C. E. (2018). The gender gap in science: how long until women are equally represented? PLoS Biology, 16(4), e2004956.

Hrabowski, F. a. (2011). Boosting minorities in science. Science, 331(6014), 125

Hurtado, S., Eagan, K., Pryor, J. H., Whang, H., \& Tran, S. (2012). Undergraduate teaching faculty: the 2010-2011 HERI faculty survey. Los Angeles: Higher Education Research Institute: University of California.

Johnson, E., Andrews-Larson, C., Keene, K., Keller, R., Fortune, N., \& Melhuish, K. Inquiry and inequity in the undergraduate mathematics classroom. Proceedings of the 40th Annual Conference of the North American Chapter of the International Group for the Psychology of Mathematics Education. Greenville, SC. In press

Madsen, A., McKagan, S. B., \& Sayre, E. C. (2013). Gender gap on concept inventories in physics: what is consistent, what is inconsistent, and what factors influence the gap? Physical Review Special Topics-Physics Education Research, 9(2), 020121 .
Maxwell, J. A. (2010). Using numbers in qualitative research. Qualitative Inquiry, 16(6), 475-482.

Meyer Monhardt, R. (2000). Fair play in science education: equal opportunities for minority students. The Clearing House: A Journal of Educational Strategies, Issues and Ideas, 74(1), 18-22.

Mickelson, R. A., Parker, A., Stearns, E., Moller, S., \& Dancy, M. (2015). Family matters: familial support and African American female success. In Ruiz-Smith \& S. L. Clark (Eds.), Contemporary African American Families: Achievements, Challenges, and Empowerment Strategies in the 21st Century. New York: Routledge-Taylor \& Francis.

Moller, S., Banerjee, N., Bottia, M. C., Stearns, E., Mickelson, R. A., Dancy, M., et al. (2015). Moving Latino/a students into STEM majors in college: the role of teachers and professional communities in secondary schools. Journal of Hispanic Higher Education, 14(1), 3-33.

National Science Foundation, National Center for Science and Engineering Statistics. (2017). Women, minorities, and persons with disabilities in science and engineering: 2017. Special Report NSF 17-310. Arlington: Available at www.nsf.gov/statistics/wmpd/.

Prince, M. (2004). Does active learning work? A review of the research. Journal of Engineering Education, 93(3), 223-231.

Rainey, K., Dancy, M., Mickelson, R., Stearns, E., \& Moller, S. (2018). Race and gender differences in how sense of belonging influences decisions to major in STEM. International Journal of STEM Education, 5(1), 10.

Seidman, I. (2013). Interviewing as qualitative research: a guide for researchers in education and the social sciences. New York: Teachers college press.

Steele, C. M., Spencer, S. J., \& Aronson, J. (2002). Contending with group image: the psychology of stereotype and social identity threat. In Advances in experimental social psychology (Vol. 34, pp. 379-440). Academic Press.

Strayhorn, T. L. (2012). College students' sense of belonging: a key to educational success for all students. New York: Routledge.

Terenzini, P. T., Cabrera, A. F., Colbeck, C. L., Parente, J. M., \& Bjorklund, S. A. (2001). Collaborative learning vs. lecture/discussion: students' reported learning gains. Journal of Engineering Education, 90(1), 123-130.

Tseng, K. H., Chang, C. C., Lou, S. J., \& Chen, W. P. (2013). Attitudes towards science, technology, engineering and mathematics (STEM) in a project-based learning (PjBL) environment. International Journal of Technology and Design Education, 23(1), 87-102.

United Nations Educational, Scientific and Cultural Organization. Women in Science : Women Sci. 2015; (34):1-4. http://uis.unesco.org/sites/default/files/ documents/fs34-women-in-science-2015-en.pdf.

Watkins, J., \& Mazur, E. (2013). Retaining students in science, technology, engineering, and mathematics (STEM) majors. Journal of College Science Teaching, 42(5), 36-41.

\section{Submit your manuscript to a SpringerOpen ${ }^{\circ}$ journal and benefit from:}

- Convenient online submission

- Rigorous peer review

- Open access: articles freely available online

High visibility within the field

- Retaining the copyright to your article

Submit your next manuscript at $\boldsymbol{\nabla}$ springeropen.com 